\title{
Auditing paediatric diabetes care and the impact of a specialist nurse trained in paediatric diabetes
}

\author{
F J Cowan, J T Warner, L M Lowes, J P Riberio, J W Gregory
}

\begin{abstract}
Aims-To define outcome measures for auditing the clinical care of children and adolescents with insulin dependent diabetes mellitus (IDDM) and to assess the benefit of appointing a dedicated paediatric trained diabetes specialist nurse (PDSN).

Methods-Retrospective analysis of medical notes and hospital records. Glycaemic control, growth, weight gain, microvascular complications, school absence, and the proportion of children undergoing an annual clinical review and diabetes education session were assessed. The effect of the appointment of a PDSN on the frequency of hospital admission, length of inpatient stay, and outpatient attendance was evaluated.
\end{abstract}

Results-Children with IDDM were of normal height and grew well for three years after diagnosis, but grew suboptimally thereafter. Weight gain was above average every year after diagnosis. Glycaemic control was poor at all ages with only $16 \%$ of children having an acceptable glycated haemoglobin. Eighty five per cent of patients underwent a formal annual clinical review, of whom $16 \%$ had background retinopathy and $20 \%$ microalbuminuria in one or more samples. After appointing the PDSN the median length of hospital stay for newly diagnosed patients decreased from five days to one day, with 10 of 24 children not admitted. None of the latter was admitted during the next year. There was no evidence of the PDSN affecting the frequency of readmission or length of stay of children with established IDDM. Non-attendance at the outpatient clinic was reduced from a median of 19 to $10 \%$.

Conclusions-Outcome measures for evaluating the care of children with IDDM can be defined and evaluated. Specialist nursing support markedly reduces the length of hospital stay of newly diagnosed patients without sacrificing the quality of care.

(Arch Dis Child 1997;77:109-114)

Keywords: diabetes education; glycaemic control; outpatient attendance; hospital admission

Correspondence to:

Dr Fiona J Cowan,

Department of Child Health,

University Hospital of Wales,

Heath Park, Cardiff CF4

4XW.

Accepted 13 May 1997
In recent years formalised audits have been widely introduced in all sections of the UK National Health Service. In clinical medicine, outcome measures provide clinicians with information about their practice which can be used to improve the delivery of patient care. In 1989 the World Health Organisation and the International Diabetes Federation produced the St Vincent declaration. ${ }^{1}$ This highlighted recommendations for standards of diabetes care, and five year targets were set for the provision of resources, ensuring the rights and responsibilities of patients, improved education, and a reduction in complications.

In Wales, the Welsh Office has produced recommendations for providing diabetes care. ${ }^{2}$ These recommendations use 'outcome measures' which relate to the process of patient education and annual review. They do not refer to the particular needs of children for whom outcome measures such as growth, school attendance, hospital admissions, clinic attendance, and glycaemic control can be used to improve patient care.

In April 1995 a dedicated paediatric trained diabetes specialist nurse (PDSN) was appointed at the University Hospital of Wales to provide education, advice, and practical support. One aim of this appointment was to enable children with newly diagnosed insulin dependent diabetes mellitus (IDDM) to start treatment at home and to facilitate early discharge of those requiring admission. Before this appointment the paediatric diabetes clinic was served by four part time diabetes nurses who worked predominantly within the adult service, most of whom had no paediatric training. These nurses were community based and were not allowed to offer a service to paediatric inpatients or outside normal working hours.

The aim of this study was to define usable outcome measures for auditing the clinical care of children with IDDM and to assess the effect of the first year of the appointment of the PDSN.

\section{Subjects and methods}

The paediatric diabetes clinic at the University Hospital of Wales provides diabetes care for children predominantly within Cardiff and the Vale of Glamorgan (total population 425800 , with 147000 aged 15 years or younger), but also serves a small proportion of the lower Rhymney, Rhondda, Cynon, and Taff valleys. At the time of the audit there were 154 children ( 81 girls and 73 boys) receiving diabetes care at the hospital. Sixteen children were less than 5 years old and 104 were older than 11 . Children are usually followed up in the clinic until 18 years of age. The median (range) duration of diabetes was $12.5(0.1-17.2)$ years. 
Table 1 Screening for complications and associated problems in children with IDDM; values are number (\%) of patients

\begin{tabular}{lccc}
\hline & Eligible for screening & Actually screened & Abnormalities detected \\
\hline Retinopathy & $87(66)$ & $78(90)$ & $12(16)$ \\
Microalbuminuria & $87(66)$ & $61(70)$ & $12(20)$ \\
Thyroid function & $154(100)$ & $109(71)$ & $2(2)$ \\
Antigliadin antibody & $154(100)$ & $109(71)$ & $4(4)$ \\
Hyperlipidaemia & $154(100)$ & $109(71)$ & $6(5)$ \\
\hline
\end{tabular}

Data on clinic attendance and glycaemic control were routinely collected and entered onto a computerised database developed by $\mathrm{Dr}$ K Robertson (Yorkhill Hospital, Glasgow) adapted for local use. Statistical analysis was carried out by appropriate parametric and non-parametric statistical methods using SPSS for Windows version 6.0 .

At the end of 1993, after a previous audit, the following standards were set for local diabetes care: all children to have an annual review; annual screening for complications and known associations of IDDM; school attendance to be documented; growth to be assessed every three months; glycaemic control to be measured every three months; children to attend outpatient clinic every three months; initiation of treatment for children with newly diagnosed IDDM to be undertaken at home where appropriate; and all children to undergo continuing education.

This audit was carried out two years after these standards were set to see if they had been achieved. The audit was subdivided to evaluate outcome measures for auditing clinical care (data collected January 1996) and to evaluate the effect of the appointment of a dedicated PDSN on hospital outpatient and inpatient attendance and on patient education. Data were collected on hospital outpatient and inpatient attendance for the two years before (1 April 1993-31 March 1995) and compared with the results for the first year after (1 April 1995-31 March 1996) the appointment of the PDSN.

OUTCOME MEASURES OF CLINICAL CARE Annual review

We intended all children with IDDM for one year or more to undergo an annual review. One hundred and twenty nine children $(84 \%)$ were eligible. A full examination was to be carried out including assessment of the hands, feet, and mouth, and the measurement of blood pressure and palpation of peripheral pulses. Pubertal development was to be assessed according to the method of Tanner. ${ }^{3}$ A limited neurological examination included testing for abnormalities of vibration and pinprick sensation and tendon reflexes. The results of the annual review were collected by retrospective analysis of the medical notes.

Complications and associated problems

Children with IDDM older than 10 years or who were five years or more from diagnosis were screened for complications of retinopathy and nephropathy. Eighty seven children (66\%) were eligible. Retinopathy was detected by fundoscopy through a dilated pupil and by retinal photography. Retinal photographs were graded for evidence of retinopathy based on the Welsh Community Diabetic Retinopathy Study, a modification of the EURODIAB grading. ${ }^{4}$ Abnormalities on either fundoscopy or retinal photography were taken as positive results. Screening for nephropathy was undertaken by collecting three early morning urine samples for the determination of microalbuminuria (assayed by nephelometry using the Beckman Array system). Urinary albumin excretion greater than $20 \mathrm{mg} / 1$ in one or more samples was taken as indicating possible significant microalbuminuria requiring further evaluation.

The prevalence of thyroid disease, coeliac disease, and hyperlipidaemia was assessed by measuring free thyroxine, thyroid stimulating hormone (assayed by automated chemiluminescence using the Chiron Diagnostics system; normal range free thyroxine $12-28 \mathrm{pmol} / \mathrm{l}$, thyroid stimulating hormone $<4.2 \mathrm{mU} / \mathrm{l}$ ), total $\operatorname{IgA}$ and $\operatorname{IgA}$ antigliadin antibodies (assayed by sandwich immunoassay using the Pharmacia CAP system; reference range $0-3 \mathrm{mg}$ antibody/l) and cholesterol and triglycerides (assayed by enzymatic colorimetric test using Sys $3 \mathrm{BM} / \mathrm{Hitachi} 747$ system; reference range for cholesterol $<5.5 \mathrm{mmol} / 1$ and triglycerides $0.6-2.0 \mathrm{mmol} / \mathrm{l})$.

\section{School attendance}

The documentation and extent of school attendance over the preceding three months was noted retrospectively from the medical records.

\section{Growth}

Height was measured by a trained auxologist using a wall mounted Harpenden stadiometer (Holtain Ltd, Crymych, Dyfed) and weight was measured using an Avery beam balance (Avery Ltd, Birmingham). Measurements were expressed as SD scores. Weight was also corrected for height (WFH\%). ${ }^{5}$ Measurements were taken from the first clinic visit after diagnosis to account for the effects of dehydration at clinical presentation and thereafter were collected for the first seven years from diagnosis.

\section{Glycaemic control}

Glycaemic control was determined by the percentage glycated haemoglobin levels $\left(\% \mathrm{HbA}_{1}\right)$ (assayed by gel electrophoresis using the Corning Glytrac method). The normal reference range for $\mathrm{HbA}_{1}$ concentrations for the nondiabetic population is $5.6-8.0$ (mean (SD) 6.8 $(0.6) \%)$. Results less than $10 \%$ for children with IDDM (5 SD above the mean nondiabetic reference level) were taken to indicate satisfactory glycaemic control. Insulin dosage in units per kilogram body weight $(\mathrm{U} / \mathrm{kg})$ was noted for each patient

\section{NON-CLINICAL OUTCOME MEASURES TO}

EVALUATE THE IMPACT OF THE PDSN

Clinic attendance

Non-attendance rates at the diabetes outpatient clinics were collected retrospectively over a three year period, two years before and one year after the appointment of the PDSN. The 


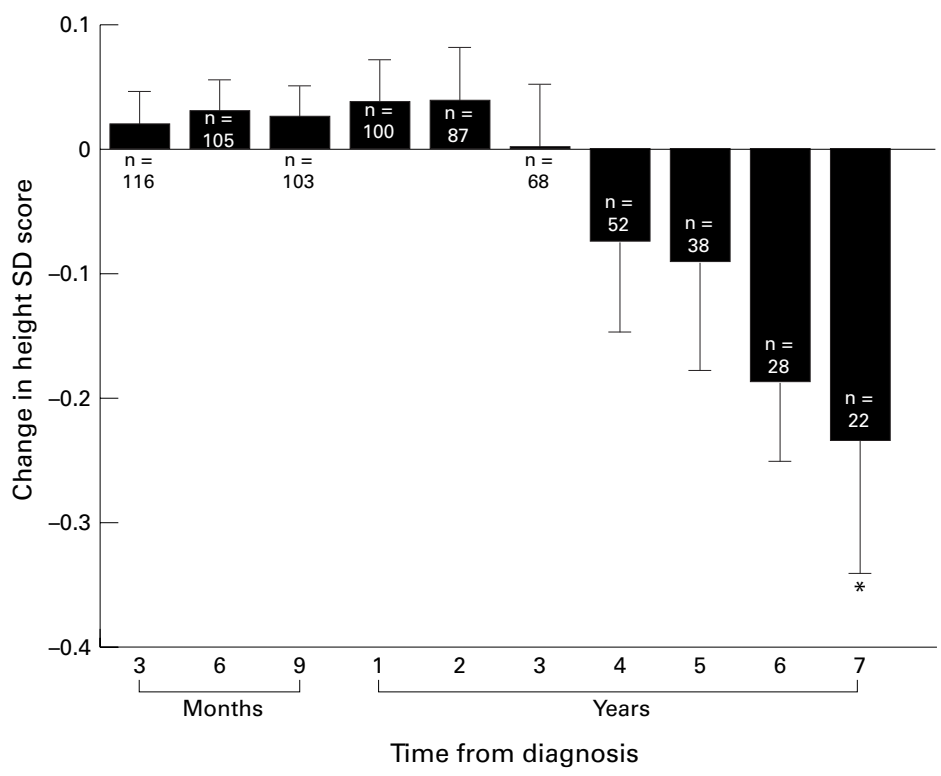

Figure 1 Mean (SE) change in height SD score from diagnosis of IDDM; ${ }^{\star} p<0.05$ compared with diagnosis.

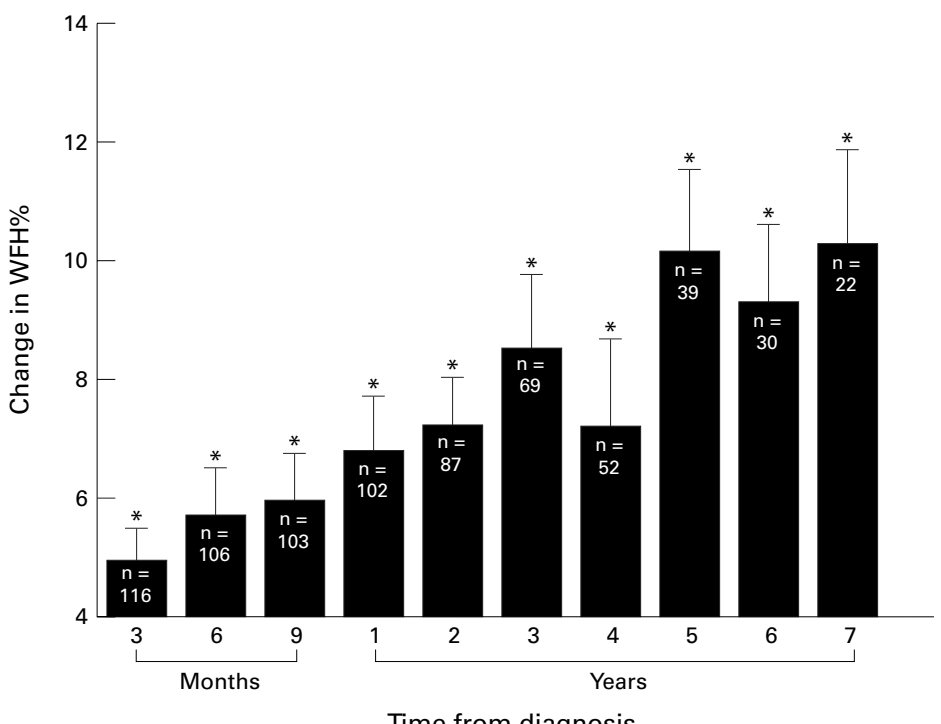

Figure 2 Mean (SE) change in WFH\% from diagnosis of IDDM; * $p<0.001$ compared with diagnosis. appointment of the PDSN. Reasons for admissions were noted.

\section{Education}

Diabetic education proformas are held by the PDSN and evidence of continuing education was expected for all children and adolescents. The education proforma covers all aspects of diabetes, including the nature of the disease, dietary advice, monitoring, hypo/ hyperglycaemia, illness, exercise, complications, and social aspects.

\section{Results}

OUTCOME MEASURES OF CLINICAL CARE

Annual review

One hundred and ten patients (85\%) had undergone an annual review, most within the 12 months before the audit. Nineteen (15\%) had never had an annual review. Of those who had been reviewed, all had their growth measured, but other data were incomplete in 19 $(17 \%)$. In half there was no comment in the notes about injection sites and one third had no documented neurological examination. Four (3\%) had increased blood pressure with one receiving treatment for hypertension. One patient had reduced vibration and pinprick sensation.

Complications and associated problems

Table 1 gives the results of the screening investigations. All the children identified with retinopathy had background retinopathy only (grade 1, that is few microaneurysms, exudates, or small haemorrhages outside the macula). No child was detected with proliferative retinopathy. Two children with abnormal thyroid function were detected: one child with subclinical autoimmune hypothyroidism and the other with hyperthyroidism. Four children were noted to have increased levels of antigliadin antibodies, of which three were normal on repeat investigation. The other child was proved to have coeliac disease.

\section{School attendance}

School attendance had been documented in $110(68 \%)$ of the medical notes. Four children had missed more than one month of school during the preceding three months. One had cystic fibrosis and three were chronic school refusers. All the other children had missed two days or less.

\section{Growth}

Growth measurements were available for 122 children at diagnosis and 105 children for at least one year after diagnosis. Long term growth data were incomplete for 32 children, mainly because of diagnosis elsewhere and subsequent transfer to our unit. These children were excluded from analysis. At diagnosis children with IDDM were of normal height with a mean (SD; range) height SD score of 0.15 $(1.07 ;-3.50$ to 2.90$)$. Growth was normal for the first three years after diagnosis, but decreased thereafter (fig 1). Children were of normal weight at diagnosis with a mean 


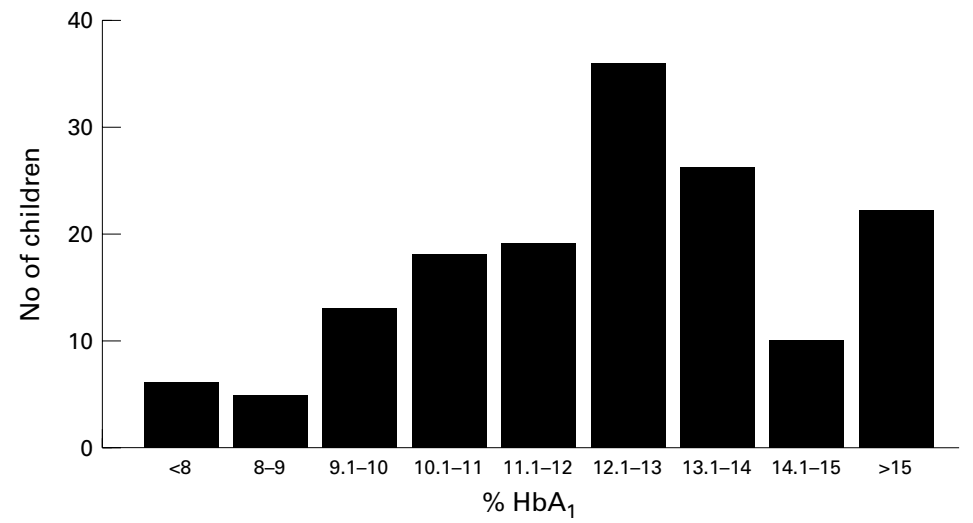

Figure 3 Distribution of percentage $\mathrm{Hb} A_{1}$ results.

$\%$ WFH of $102 \%$. Weight increased significantly in the seven years after diagnosis $(\mathrm{p}<0.001)$ (fig 2).

\section{Glycaemic control}

The mean (SD) percentage $\mathrm{HbA}_{1}$ for all children was 12.5 (2.5). Children older than 11 years had a significantly higher mean (SD) percentage $\mathrm{HbA}_{1}$ than those less than this age (12.7 (2.5)\% and 11.7 (2.1)\%, respectively, $\mathrm{p}<0.05)$. A total of 129 children $(84 \%)$ had an $\mathrm{HbA}_{1}$ greater than $10 \%$, higher than the ideal target range (fig 3 ).

The mean dose of insulin was $0.8 \mathrm{U} / \mathrm{kg}$ (range $0.1-2.0 \mathrm{U} / \mathrm{kg}$ ). There was a significant association between insulin dose and glycaemic control; those treated with the highest doses of insulin had the worst control $(\mathrm{p}<0.05)$. There was no relation between insulin dose and growth or adiposity.

NON-CLINICAL OUTCOME MEASURES TO EVALUATE THE IMPACT OF THE PDSN

Clinic attendance

Table 2 shows the attendance rates at clinics in the two years before and one year after the appointment of the specialist nurse. A significant reduction in non-attendance rates was seen in both clinics, but more notably in the clinic for younger children.

\section{Hospital admissions}

In the two years before appointing the PDSN the median (range) length of stay in hospital after diagnosis was five (2-18) days, and after the appointment one (0-7) day(s) (fig 4). Ten of the 24 children presenting in the year after the PDSN appointment did not need admission; before this appointment all children were admitted after diagnosis. No child who started insulin treatment at home was readmitted.

No change has yet been seen in the rate of readmission or length of stay of children

Table 2 Median (range) non-attendance rates (\%) at the diabetes clinic

\begin{tabular}{lll}
\hline Year & Paediatric clinic & Adolescent clinic \\
\hline 1 & $21(0-42)$ & $31(12-55)$ \\
2 & $19(0-44)$ & $38(25-59)$ \\
$3 \dagger$ & $10(0-43)^{\star}$ & $23(7-56)^{\star \star}$ \\
\hline
\end{tabular}

† After appointment of PDSN.

$\star \mathrm{p}<0.001 ;{ }^{\star \star} \mathrm{p}<0.05$ for year 3 compared with years 1 and 2 .

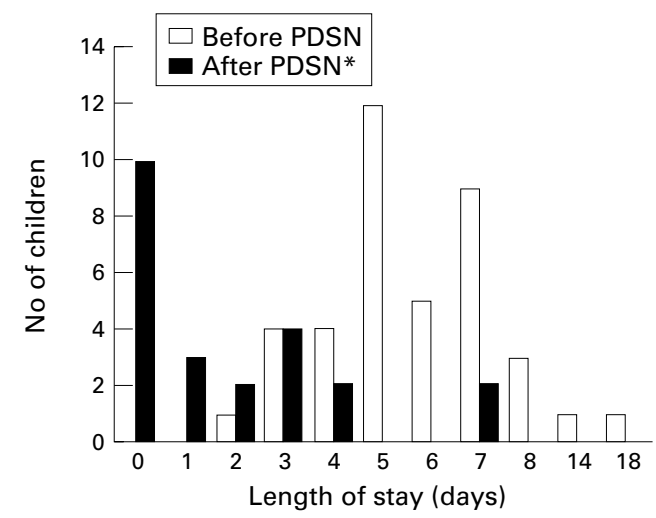

Figure 4 Length of hospital stay for patients with newly diagnosed IDDM in relation to the appointment of a PDSN; ${ }^{\star} p<0.001$.

diagnosed with IDDM before the appointment of the PDSN. Twenty eight such children each year require readmission, with a median (range) length of stay of two (0.5-13) days. Most of these admissions are due to ketoacidosis.

\section{Education}

All patients had an individual diabetic educational proforma and 144 children (94\%) underwent continuing diabetic education either at home or in the clinic.

\section{Discussion}

It has been suggested that the appropriate outcome measures in contracting for diabetes care should be that patient education is continuing and that patients undergo an annual review. ${ }^{2}$ These processes, rather than outcome measures, have been recommended because other variables, such as those used in this study, may not reflect the contracting process as they are not directly under the control of doctors but require patient cooperation. However, patient compliance may partly be a measure of the success of education and therefore we believe that growth, glycaemic control, hospital admission, and clinic visits are valid outcome measures for audit.

This study has shown, along with others, ${ }^{7}$ that children grow well in the first three years after diagnosis, but thereafter growth deteriorates. It is postulated that this may be due to a blunting or delay in the pubertal growth spurt which is known to occur, particularly in diabetic girls. In our audit specific details on stages of puberty were not collected so we cannot comment on whether pubertal delay may be contributing to the reduction in height SD scores with time from diagnosis. The fall off in growth did not appear to be a temporary phenomenon, however, as would be expected if delayed puberty were the cause, as the children who had reached final height had a mean (SD; range) final height SD score of -0.54 (1.2; -3.5 to 1.2 ). It might be expected that children grow well initially from diagnosis due to the anabolic effects of insulin in those who were previously insulin deficient. The fall off in growth three years from diagnosis may reflect the influence of deteriorating glycaemic control in that poor control is known to be associated 
with poor growth. ${ }^{7-9}$ The continued excess weight gain observed in this study is of concern as a risk factor for the later development of ischaemic heart disease and possibly diabetic retinopathy. ${ }^{10}$

The Diabetes Control and Complications Trial has shown that improved glycaemic control has a notable benefit in reducing the complications of IDDM. ${ }^{11}$ Microvascular complications are rarely seen in prepubertal children, hence the restriction of our screening to children over 10 years of age or with IDDM for more than five years. Complications are known to be related to poor long term glycaemic control. ${ }^{12}$ Although glycated haemoglobin concentrations less than $4 \mathrm{SD}$ above the mean laboratory specific reference levels have been taken to indicate satisfactory glycaemic control, ${ }^{13}$ there is debate regarding this. The British Diabetic Association has recommended aiming for much tighter control. ${ }^{14}$ At the time of our audit we took $\mathrm{HbA}_{1}$ concentrations less than $10 \%$ to indicate satisfactory control, which equates to values within $5 \mathrm{SD}$ of the mean non-diabetic reference range. Glycaemic control was worse for children older than 11 years, adolescence being a notoriously difficult age at which to achieve good control. ${ }^{15}$ The results for our clinic were disappointing and indicated poor glycaemic control overall compared with the target, with $84 \%$ of the children having $\mathrm{HbA}_{1}$ values greater than $10 \%$. This might suggest that the 'ideal' target value is unrealistic and comparative studies between different clinics would help clarify this. Nevertheless, the finding of background diabetic retinopathy in $16 \%$ of children in our clinic suggests a measurable consequence of poor glycaemic control. This finding also confirms the importance of a detailed skilled retinal examination by appropriately trained staff. Others have also described a high incidence of background retinopathy after detailed examination. ${ }^{16}$

Few associated problems of IDDM were detected at the annual review. One child was diagnosed with coeliac disease, identified by positive antigliadin antibodies and confirmed by taking a jejunal biopsy sample. Detecting six children with hyperlipidaemia allowed dietary advice to be given with the aim of reducing the impact of this risk factor on later cardiovascular complications.

We took a urinary albumin excretion of greater than $20 \mathrm{mg} / \mathrm{l}$ in one sample to represent possible microalbuminuria. This might be considered a low threshold and could give rise to an artificially high rate of microalbuminuria in our clinic. Positive screening stimulated further investigation of the patients identified, initially by collecting a further three urine samples. Although this is non-invasive we accept that it might cause unnecessary anxiety and taking a positive result in two samples rather than just one might be preferable. We are concerned that one third of children did not have a documented neurological examination. Others have found a significant number of children to have evidence of subclinical neu- ropathy and if no appropriate examination is carried out these will be missed. ${ }^{17} 18$

The appointment of a dedicated PDSN might have been expected to improve education, clinic attendance, and hospital admissions, these being the main aims. The PDSN has a major role in the continuing education of the children with IDDM and their families and there is growing evidence that effective education programmes can reduce complications. ${ }^{19}$ One year after this appointment we have shown improvements in care. The non-attendance rate at clinic visits for the children's clinic improved from 19 to $10 \%$ and for the adolescent clinic from 38 to $23 \%$. This is not unexpected as adolescents with IDDM are more difficult to motivate. The most notable effect of the appointment of the specialist nurse has been to reduce the admission rates and length of stay for newly diagnosed patients with IDDM. Thus the median hospital stay at diagnosis has fallen from five days to one day, with 10 children requiring no admission. None of these children subsequently required readmission in the year after the appointment. Before the PDSN appointment all children were admitted at diagnosis. There was no difference in the ages of the children diagnosed with IDDM in the two years before and the year after the appointment of the PDSN. In keeping with others ${ }^{20}$ we have met no adverse effects of this early discharge and the children and their parents have all expressed satisfaction. There are considerable advantages in initial home based management: the distress of admission is avoided and the child and parents are often more at ease. So far the PDSN has had no effect on admissions of children with established diabetes, but this might be expected to take longer as established views and behaviour are less amenable to change.

\section{Recommendations}

This audit of paediatric diabetes care has led to several recommendations and targets for the next three years to improve outcome.

- Continue annual review: $90 \%$ of eligible children within any 12 month period and documentation of inspection of injection sites and neurological examination to be improved to $100 \%$

- Reduce adolescent outpatient nonattendance to $10 \%$

- Improve glycaemic control: $2 \%$ improvement in $\mathrm{HbA}_{1}$ across the clinic population

- Retinopathy and microalbuminuria screening to continue: improve microalbuminuria screening to $90 \%$ of those eligible and maintain retinopathy screening at $90 \%$

- Reduce prevalence of microalbuminuria by one third to $14 \%$

- Reduce prevalence of retinopathy by one third to $12 \%$

- Continue annual measurement of serum lipids and referral to dieticians of all those subjects with abnormally high concentrations

- Reduce by one third readmissions of children with IDDM.

We intend to use the dedicated PDSN further to help achieve these recommendations. A 24 
hour telephone advice service has been set up to deal with alterations in insulin treatment and give general advice on diabetic management. We hope that this instant response service will enable children whom we currently admit when unwell to be managed at home. Reminders of imminent clinic appointments will be sent to attempt to reduce non-attendance rates. Improving glycaemic control remains the greatest challenge. We need to convince our teenagers, through targeted education, that better control will be beneficial in the long term. With this aim we are setting up age banded education sessions. The PDSN will make home visits to follow up poor $\mathrm{HbA}_{1}$ results and give personal advice on how improvements can be made. This will enable closer monitoring than simply giving advice at clinic visits every three months. If improvements in control can be achieved, then we would expect the frequency of complications to be reduced. Emphasis on the importance of the annual review will be given to the clinic doctors to achieve our aim of a $90 \%$ uptake.

We are grateful to Dr D Owens for providing the retinopathy screening results. We are grateful to Lilly Pharmaceuticals and South Glamorgan Health Authority for 'pump priming' the initial funding for the PDSN

1 Workshop Report. Diabetes care and research in Europe: the Saint Vincent Declaration. Diabetic Med 1990;7:360.

2 Welsh Office Health Department. Contracting for health diabetes mellitus. Cardiff: Strategic Management Division, Welsh Office Health Department, 1994.

3 Tanner JM. Growth at adolescence. 2nd Ed. Oxford: Blackwell, 1962.

4 Aldington SJ. Methodology for retinal photography and assessment of diabetic retinopathy. Diabetologia 1995;38 437-44.

5 Tanner JM, Whitehouse RH, Takaishi M. Standards from birth to maturity for height, weight, height velocity and weight velocity: British children, 1965. Part I. Arch Dis Child 1966;41:454-71.
6 Tanner JM, Whitehouse RH, Takaishi M. Standards from birth to maturity for height, weight, height velocity and weight velocity: British children, 1965. Part II. Arch Dis Child 1966;41:613-35.

7 Brown M, Ahmed ML, Clayton KL, Dunger DB. Growth during childhood and final height in type 1 diabetes. Diabetic Med 1994;11:182-7.

8 Clarke WL, Vance ML, Rogol AD. Growth and the child with diabetes mellitus. Diabetes Care 1993;16:101-6.

9 Jivani SKM, Rayner PHGW. Does control influence the growth of diabetic children. Arch Dis Child 1973;48:10915 .

10 Segal N. Obesity as a risk factor in diabetic retinopathy. Oftalmologia 1992;36:35-8.

11 Diabetes Control and Complications Trial Research Group. The effect of intensive treatment of diabetes on the development and progression of long-term complications in insulin-dependent diabetes mellitus. $N$ Engl $f \mathrm{Med}$ 1993;329:977-86.

12 Chase HP, Jackson WE, Hoops SL, Cockerham RS, Archer PG, O'Brien D. Glucose control and the renal and retinal complications of insulin dependent diabetes. $\mathcal{F} A M A 1989$; 261:1155-60.

13 Komulainen J, Lounamaa R, Knip M, Kaprio EA, Åkerblom $\mathrm{HK}$ and the Childhood Diabetes in Finland Study Group. Ketoacidosis at the diagnosis of type 1 (insulin dependent) diabetes mellitus is related to poor residual cell function. Arch Dis Child 1996;75:410-5.

14 Diabetes Service Advisory Committee and British Diabetic Association. Recommendations for the management of diabetes in primary care. London: Diabetes Service Advisory Committee and British Diabetic Association, 1993.

15 Robinson JJA, Sowden JA, Tattersall RB. The management of diabetes in adolescents and young adults: a preliminary case study. F Clin Nurs 1995;4:257-65.

16 Bonney M, Hing SJ, Fung AT, et al. Development and progression of diabetic retinopathy: adolescents at risk. Diabetic Med 1995;12:967-73.

17 Olsen BS, Nir M, Kjaer I, Volund A, Mortensen HB. Elevated vibration perception threshold in young patients with type 1 diabetes in comparison to non-diabetic children and adolescents. Diabetic Med 1994;11:888-92.

18 Donaghue KC, Fung AT, Fairchild JM, Howard NJ, Silink M. Prospective assessment of autonomic and peripheral nerve function in adolescents with diabetes. Diabetic Med nerve function

19 Siddons H, McAughey D. Professional development brings specialist knowledge. The role of the diabetes specialist nurse: the Manchester model. Prof Nurse 1992;7:321-4.

20 Swift PG, Hearnshaw JR, Botha JL, Wright G, Raymond NT, Jamieson KF. A decade of diabetes: keeping children out of hospital. BMF 1993;307:96-8. 